\title{
VALORACIÓN PRESCRIPTIVA HACIA LAS ESPECIALIDADES FARMACÉUTICAS GENÉRICAS (EFG) POR MÉDICOS CONTRATADOS EN FARMACIAS DE VILLAHERMOSA
}

Heberto Romeo Priego Álvarez," Juan Antonio Córdova Hernández," María Isabel Ávalos García*, Juan Carlos López Gómez**

\begin{abstract}
Priego-Álvarez H.R., Córdova-Hernández J.A., ÁvalosGarcía M.A., López-Gómez J.C. Valoración prescriptiva hacia las Especialidades Farmacéuticas Genéricas (EFG) por médicos contratados en farmacias de Villahermosa.Hitos de Ciencias Económico Administrativas 2014;20 (57): 69-74.
\end{abstract}

\section{RESUMEN}

Objetivo: Analizar la percepción y actuación de médicos contratados por farmacias en la prescripción de medicamentos genéricos en Villahermosa, Tabasco.

Material y Método: Investigación descriptiva transversal observacional. Se efectuó un muestreo no probabilístico de conveniencia dada las características de cada cadena farmacéutica (ubicación geográfica, número de sucursales y médicos en consultorios) con una $\mathrm{n}=132$ médicos. La información se recabó durante los meses de julio a septiembre del 2013 mediante la aplicación de un cuestionario mixto. El procesamiento y análisis de datos se efectuó en el programa SPSS (Statistical Package for Social Sciences) versión 19 con estadística descriptiva y tablas de frecuencia.

Resultados: El perfil de los médicos contratados por farmacia corresponden mayormente a varones jóvenes (entre 25 y 36 años de edad), con estudios solo de licenciatura sin certificación médica y con antigüedad laboral menor a 5 años. Se consideraron 4 factores o componentes de la valoración prescriptiva: El factor conocimiento (El 84\% consideran que el medicamento genérico es bioequivalente, consigue efectos clínicos similares y tiene las mismas garantías de calidad que el medicamento original, además de que su uso no limita la libertad de prescripción). Factor ahorro o disminución del gasto farmacia (52\%), el factor competencia profesional (la prescripción del médico no está mayoritariamente orientada hacia las EFG) el factor actitud legal comercial (87\% estimó que la comercialización de las EFG es legal).
Priego-Álvarez H.R., Córdova-Hernández J.A., ÁvalosGarcía M.A., López-Gómez J.C. Prescriptive assessment towards the generic pharmaceutical specialties through physicians hired by pharmacies in Villahermosa. Hitos de Ciencias Económico Administrativas 2014;20 (57): 69-74.

\section{ABSTRACT}

Objective: Analyze the perception and performance of physicians hired by pharmacies in relation to the prescription of generic medicines in Villahermosa, Tabasco.

Material and Method: Cross-sectional observational descriptive research. ANon-probabilistic convenience sample was performed given the characteristics of each pharmacy chain (geographical location, number of branches and doctors' offices) with an $n=132$ physicians. The information was collected from July to September 2013 by applying a mixed questionnaire. The processing and analysis of the data was held with the SPSS program (Statistical Package for Social Sciences) version 19 with descriptive statistics and frequency tables.

Results: The profile of the physicians hired by each pharmacy corresponds mostly to young males (between 25 to 36 years old), with only undergraduate studies without medical certification and job seniority less than 5 years. Four factors or components of prescriptive assessment were considered: The Knowledge factor (84\% consider that the generic drug is bioequivalent, achieves similar clinical effects, and has the same quality degree as the original drug, in addition that its use does not limit the freedom of prescription). The Saving Factor or Reduced Pharmacy Expenditure (52\%), the Professional Competence Factor (doctor's prescription is not primarily oriented towards the generic pharmaceutical specialties, the Commercial Legal Attitude Factor $(87 \%$ estimated that the commercialization of the generic pharmaceutical specialties is legal).

\footnotetext{
* Profesores investigadores de la División Académica de Ciencias de la Salud de la Universidad Juárez Autónoma de Tabasco.

"Estudiante de la Licenciatura de Médico Cirujano, DACS - UJAT.
} 
Conclusiones: Los médicos consideran que las Especialidades Farmacéuticas Genéricas (EFG) tienen la misma eficacia terapéutica, bioequivalencia y biodisponibilidad que los medicamentos de patente. La opinión de los médicos frente al probable ahorro derivado de usar las EFG no es contundente; sin embargo, respecto al género son las profesionales mujeres las que están más a favor de la contención del gasto farmacéutico por su uso.
Conclusions: Physicians consider that the Generic Pharmaceutical Specialties have the same therapeutic efficacy, bioequivalence and bioavailability as the patent drugs. The medical opinion against the probable savings derived from using the generic pharmaceutical specialties is not conclusive, however, regarding the gender, the women- doctors are mostly those who are in favor to the contention of the pharmaceutical expenditure for its use.
Palabras clave: Prescripción médica. Medicamentos genéricos. Farmacias.
Key words: Medical Prescription. Generic Drugs. Pharmacies.

DIRECCIÓN PARA RECIBIR CORRESPONDENCIA:Correo electrónico: heberto_priego@hotmail.com

$\mathbf{U}$

na definición amplia de Medicamentos Genéricos o Especialidades Farmacéuticas Genéricas (EFG) aceptada globalmente es la de ser «un producto farmacéutico cuya patente ha caducado y puede ser fabricado y suministrado por otras compañías además de la poseedora hasta entonces de la patente." Dado que no se pagan royalties (regalías) al original, dichos medicamentos pueden ser comercializados a menor costo, lo que representa un ahorro tanto para el consumidor como para las instituciones de salud que las consumen.

El mercado libre de EFG es aquel en el que la compra o adquisición del fármaco, se da de manera directa por los compradores en los puntos de venta (farmacias) y obedece a una transacción económica. Existe otro mercado con características de obligatoriedad que es cuando se proporciona EFC al consumidor por una institución de salud determinada (seguridad social) sin pago directo. En esta última situación, la contención del gasto farmacéutico es debida a la compra consolidada de medicamentos genéricos, política comúnmente aceptada y bien valorada en nuestro país. Otro particular aspecto a considerar es el aumento de los precios de los fármacos al público y la contribución que al ahorro de la economía familiar puede representar el consumo de EFG. Al respecto cabe apuntar que en Tabasco de acuerdo con información del Instituto Nacional de Estadística y Geografía (INEGI), en los últimos seis años el costo de los medicamentos ha registrado un aumento de precios de 28.71 por ciento en promedio. Los datos del INEGI señalan que el incremento promedio de la canasta básica en el subíndice de medicamentos, ha mostrado un comportamiento constante a la alza de 2007 al presente año, por lo que nos hace pensar que si hay más prescripción de EFG el paciente se beneficia al reducir sus gastos. A nivel federal, la secretaria de salud, Dra. Mercedes Juan, anunció recientemente la aprobación de 33 nuevos genéricos que se utilizan para prevenir y tratar diversas enfermedades crónicas no transmisibles como hipertensión, diabetes y cáncer, entre otras. Con estas 33 versiones genéricas suman ya 214 los fármacos -de 27 sustancias activas que perdieron su patente- autorizados en el marco de la Estrategia de Liberación de Genéricos para el Ahorro de las Familias Mexicanas implementada por el actual gobierno federal (El Economista, 2013).

Las cadenas de farmacias con servicios de atención médica son un mercado creciente, ya que de acuerdo con la consultora IMS Health, actualmente existe 10 mil médicos establecidos en las principales cadenas, por ejemplo Farmacias del ahorro, Farmacias de similares, y Farmacias Benavides. Cada médico atiende en promedio 25 pacientes por día; es decir, 250 mil consultas diarias en total por toda la red de consultorios auspiciados por las cadenas de farmacias. La colocación de médicos privados en las cadenas de farmacias, creó un sistema alterno de atención primaria de la salud pues estos doctores dan 250 mil consultas todos los días, casi el mismo número que ofrece el Instituto Mexicano del Seguro Social (IMSS) y tres veces las que otorga el Instituto de Seguridad Social al Servicio de los Trabajadores del Estado (Agencia Reforma, 2013). 
En México; es poco lo que se conoce respecto a la percepción y actuación de los médicos prescriptores, en el mercado libre de EFG, por lo que el análisis de su consumo sería de gran utilidad para normar las políticas del sector, estableciendo las bases para las campañas de sensibilización para mediadores del proceso de compra (médicos prescriptores), (UNED, 2010).

El objetivo central del presente trabajo es analizar la percepción y actuación de médicos contratados por farmacias en la prescripción de medicamentos genéricos en Villahermosa, Tabasco.

\section{Material y Método}

El estudio realizado es de tipo descriptivo transversal observacional. Para la selección de los informantes se efectuó un muestreo no probabilístico de conveniencia dada las características de cada cadena farmacéutica (ubicación geográfica, número de sucursales y médicos en consultorios). Para el estudio se consideraron 43 farmacias existentes en 4 zonas geográficas de Villahermosa: 1) Centro, 2) Águila 1‥ de Mayo, 3) Atasta, y 4) Tamulté de las Barrancas (4 de Farmacias Unión, 12 de Farmacia del Ahorro, 25 de Fundación Best, y 2 de Farmacia del Dr. Descuento). La información se recabó durante los meses de julio a septiembre del 2013, con una $n=132$ médicos.

Para la recolección de los datos se partió del instrumento elaborado por A.J. García y cols. del Departamento de Farmacología de la Facultad de Medicina de la Universidad de Málaga en España (2003). Dicho instrumento: PAMPEFG-01 (Percepción y Actuación de Médicos en la Prescripción de Especialidades Farmacéuticas Genéricas), fue ligeramente modificado para estar acorde con el objetivo de la investigación, el cual consideró 27 reactivos agrupados en tres apartados:

Tabla 1
I. Sociodemográficos, II. Percepción y actuación del médico, y III. Prescripción de medicamentos. Para su aplicación y recolección de los datos se contó con el apoyo de dos encuestadores a quienes previamente se les capacitó.

El procesamiento y análisis de datos se efectuo en el programa SPSS (Statistical Package for Social Sciences) versión 19 con estadística descriptiva y tablas de frecuencia.

\section{Resultados}

La información se obtuvo de 132 médicos generales con contrato vigente en 5 cadenas de farmacias con consultorios (8 médicos de Farmacia Unión, 28 de la Farmacia del Ahorro, 90 de la Fundación Best, y 6 de Farmacia del Dr. Descuento).

Caracterización de los médicos prescriptores contratados en cadenas farmacéuticas

\begin{tabular}{|c|c|c|}
\hline Características & $n=132$ & Porcentaje (\%) \\
\hline \multicolumn{3}{|l|}{ Género } \\
\hline Masculino & 79 & 60 \\
\hline Femenino & 53 & 40 \\
\hline \multicolumn{3}{|l|}{ Grupo de edad } \\
\hline $25-28$ & 40 & 30 \\
\hline $29-32$ & 35 & 27 \\
\hline $33-36$ & 27 & 21 \\
\hline $37-40$ & 17 & 13 \\
\hline $41-44$ & 6 & 5 \\
\hline $45-48$ & 3 & 2 \\
\hline $49-52$ & 3 & 2 \\
\hline $53-56$ & 1 & 1 \\
\hline \multicolumn{3}{|l|}{ Formación Profesional } \\
\hline Médicos Generales & 132 & 100 \\
\hline \multicolumn{3}{|l|}{ Certificación Medica } \\
\hline Tienen & 55 & 60 \\
\hline No tienen & 77 & 40 \\
\hline \multicolumn{3}{|l|}{ Antigüedad Laboral } \\
\hline$<1$ año & 14 & 11 \\
\hline $1-5$ años & 60 & 46 \\
\hline$>5-10$ años & 34 & 26 \\
\hline$>10$ años & 24 & 18 \\
\hline
\end{tabular}


El perfil de los medicos contratados por farmacia correspondende mayormente a varones jóvenes (entre 25 y 36 años de edad), con estudios solo de licenciatura sin certificación médica y con antigüedad laboral menor a 5 años (ver tabla 1).

En la tabla 2 se exponen las opiniones de los médicos encuestados acerca de los diferentes ítems en una escala tipo Likert con 5 respuestas posibles.

Con respecto a los factores que intervienen en la valoración prescriptiva de los médicos contratados por las farmacias, se consideraron 4 factores 0 componentes de las opiniones (ver tabla 3). El factor conocimiento (ítems 1-6) respecto a qué saben los médicos sobre las EFG, reflejó que la mayoría (84\%) consideran que el medicamento genérico es bioequivalente, consigue efectos clínicos similares y tiene las mismas garantías de calidad que el medicamento original, además de que su uso no limita la libertad de prescripción. En el Factor Ahorro o disminución del gasto farmacia (ítems 7 -8) que hace referencia a las opiniones de los médicos sobre posibles ahorros económicos derivados de la utilización y uso de las EFG; es decir, hasta qué punto pueden contribuir a la contención del gasto farmacéutico desde su punto de vista profesional y laboral no fue contundente, dado que solo un poco más de la mitad estuvo a favor (52\%); sin embargo, respecto al género son las mujeres quienes están más a favor de la reducción del gasto

Tabla 2. Valoración prescriptiva de los médicos contratados por farmacias

\begin{tabular}{|c|c|c|c|c|c|}
\hline ÍTEMS & $\begin{array}{l}\text { TD } \\
\%\end{array}$ & $\begin{array}{l}\text { ED } \\
\%\end{array}$ & I & $\begin{array}{l}\text { DA } \\
\%\end{array}$ & $\begin{array}{l}\text { TA } \\
\%\end{array}$ \\
\hline $\begin{array}{l}\text { 1. Las EFG tienen la misma bioequivalencia y biodisponibilidad que los medicamentos } \\
\text { de patente. }\end{array}$ & 6 & .0 & 1 & $\underline{51}$ & 42 \\
\hline $\begin{array}{l}\text { 2. Las EFG consiguen el mismo efecto clínico que los medicamentos de patente } \\
\text { (original de referencia). }\end{array}$ & 4 & 8 & .0 & $\underline{48}$ & 40 \\
\hline 3. Generalmente las EFG no cumplen con el efecto terapéutico (tratamientos fallidos). & 45 & $\underline{54}$ & .0 & 1 & .0 \\
\hline 4. El uso de las EFG aumentan el riesgo de efectos secundarios. & 44 & $\underline{54}$ & .0 & 1 & 1 \\
\hline $\begin{array}{l}\text { 5. La EFG debe de ser esencialmente similar al medicamento original de referencia } \\
\text { (medicamento de patente). }\end{array}$ & 1 & .0 & 8 & 45 & $\underline{46}$ \\
\hline $\begin{array}{l}\text { 6. Un EFG ofrece las mismas garantías de calidad del preparado por una marca } \\
\text { comercial. }\end{array}$ & 1 & 1 & .0 & $\underline{51}$ & 47 \\
\hline 7. La prescripción de EFG disminuye el gasto farmacéutico en atención primaria. & .0 & .0 & .0 & $\underline{52}$ & 48 \\
\hline $\begin{array}{l}\text { 8. Las EFG deben usarse en sustitución de los medicamentos de patente por su bajo } \\
\text { costo. }\end{array}$ & .0 & .0 & .0 & $\underline{52}$ & 48 \\
\hline $\begin{array}{l}\text { 9. Normalmente receto medicamentos genéricos (Especialidades Farmacéutica } \\
\text { Genérica). }\end{array}$ & .0 & .0 & .0 & $\underline{52}$ & 48 \\
\hline 10. En mi centro de trabajo se prioriza la prescripción de las EFG. & .0 & .0 & .0 & $\underline{52}$ & 48 \\
\hline $\begin{array}{l}\text { 11. El uso de EFG disminuye la capacidad de elección del médico en la prescripción de } \\
\text { medicamentos. }\end{array}$ & $\underline{52}$ & 48 & .0 & .0 & .0 \\
\hline 12. Los médicos suelen obtener regalías por recetar medicamentos comerciales. & 47 & $\underline{52}$ & .0 & .0 & 1 \\
\hline 13. Se desconoce la calidad de los laboratorios que fabrican las EFG. & $\underline{\mathbf{5 1}}$ & 48 & .0 & 1 & .0 \\
\hline 14. La mayoría de las EFG que existen en el mercado son piratas (copia ilegal). & 50 & 50 & .0 & .0 & .0 \\
\hline 15. El uso de las EFG limita la creación e investigación de nuevas patentes. & $\underline{47}$ & 45 & 7 & 1 & .0 \\
\hline 16. Los pacientes valoran más los medicamentos de patente que las EFG . & .0 & .0 & .0 & $\underline{52}$ & 48 \\
\hline 17. Las EFG se encuentran disponibles en farmacias e instituciones de salud. & .0 & .0 & .0 & 47 & $\underline{53}$ \\
\hline $\begin{array}{l}\text { 18. El farmacéutico (o dispensador de farmacia) no puede decidir la venta o } \\
\text { surtimiento de un fármaco entre una marca comercial o una genérica, a pesar de } \\
\text { haber sido recetado por su composición genérica. }\end{array}$ & 8 & 3 & 6 & 36 & $\underline{47}$ \\
\hline
\end{tabular}

NOTA: Totalmente en desacuerdo (TD), En desacuerdo (ED), Indeciso (I), De acuerdo (DA) y Totalmente de Acuerdo (TA) 
farmacéutico por su uso. En el bloque Factor competencia profesional (ítems 9-12) se estudian las opiniones de los médicos acerca de su actitud frente a la prescripción y dispensación de los medicamentos genéricos, siendo la principal concordancia la valoración de que la prescripción del médico no está mayoritariamente orientada hacia las EFG, ya que tienen la misma posibilidad de prescribir un medicamento comercial que de uno genérico, y el Factor actitud legal comercial (ítems 13-18) en el que se analizan las actitudes (en cuanto a esencia, forma y consideración) de los médicos en relación con las EFG, la opinión mayoritaria (87\%) estimó que la comercialización de las EFG es legal, que no existen copias ilegales en el mercado nacional, que los medicamentos genéricos no son impedimento para la investigación y creación de nuevas patentes y se remarcó que tienen los mismos efectos terapéuticos que los originales.

\section{Discusión}

El estudio describe un perfil laboral de los médicos contratados por farmacias: en su mayoria son generalistas (médicos generales) jóvenes (entre 25 a 32 años) mayoritariamente hombres, con poca experiencia profesional (menor a 5 años) y sin certificación profesional. Lo cual es explicable a los bajos salarios que ofrecen las cadenas farmacéuticas y a la baja seguridad laboral de su contratación.
Estos médicos generales que trabajan en las farmacias, de acuerdo con la encuesta aplicada, están a favor de los medicamentos genéricos en cuanto a su eficacia terapéutica, bioequivalencia y biodisponibilidad. En este sentido, la calidad de los medicamentos genéricos está garantizada por toda la documentación técnica y administrativa que exigen las autoridades sanitarias de cada país para su autorización y registro. El hecho de que la juventud sea una característica de mayor nivel de conocimiento hacia las EFG es coincidente con el estudio realizado en Málaga, España (García, A. 2003).

A diferencia de otros estudios (García, A.; Isurralde 2007) donde la respuesta sobre si los medicamentos génericos limitan la libertad de prescripción fue ambigua, los médicos de farmacias investigados no identificaron a las EFG como elementos de restricción. La leyes de salud mexicanas establecen la prescripción con base al principio activo del fármaco, siendo una alternativa neutra de prescripción que impide la expedición de recetas por marcas comerciales. Ahora bien, dada las características de contratación de estos médicos, cabría suponer que políticas internas orienten hacia la venta de los productos génericos propios.

\section{Conclusiones}

Los médicos contratados por farmacias tienen como perfil laboral ser generalistas (médicos generales) jóvenes (entre 25 a 32 años) mayoritariamente hombres, con poca experiencia profesional (menor a 5 años) y sin certificación profesional.

Tabla 3. Factores intervinientes en la valoración prescriptiva de los médicos contratados por farmacias.

\begin{tabular}{lccc}
\hline \multicolumn{1}{c}{ Variable } & Ítems & Valoración & Opinión \\
\hline Factor Conocimiento & $1-6$ & $\mathbf{8 4 \%}$ & De acuerdo \\
& & $(111)$ & \\
Factor Ahorro (Disminución del & $7-8$ & $\mathbf{5 2 \%}$ & De acuerdo \\
Gasto Farmacia) & & $(68)$ & \\
Factor Competencia Profesional & $9-12$ & $\mathbf{4 9 \%}$ & De acuerdo \\
& & $(68)$ & \\
Factor Legal-Comercial & $13-18$ & $\mathbf{8 7 \%}$ & De acuerdo \\
& & $(117)$ & \\
\hline
\end{tabular}


Estos médicos consideran que las EFG tienen la misma bioequivalencia y biodisponibilidad, consiguen el mismo efecto terapéutico que los medicamentos de patente, y que tienen las mismas garantías de calidad que el preparado que una marca comercial.

La opinión de los médicos frente al probable ahorro derivado de usar las EFG no es contundente; sin embargo, respecto al género son las profesionales mujeres las que están más a favor de la contención del gasto farmacéutico por su uso. Opinan mayoritariamente que la comercialización de las EFG es legal, que no existen copias ilegales en el mercado nacional, que no son impedimento para la investigación y creación de nuevas patentes.

\section{REFERENCIAS}

A.J. García, F. Martos, F. Leiva, F. Sánchez, (2003). Genéricos: ¿Buenos omalos? Conocimientos y actitudes de los médicos ante los medicamentos genéricos. Gaceta Sanitaria 17(2), pp.144-149.

Insaurralde, M., Grandolio, P., Gómez, M., Servin, R. (2006). Evaluación de los conocimientos y opiniones de médicos ante la prescripción de medicamentos genéricos en las provincias de Chaco y Corrientes. Hospital «Dr. Julio Cecilio Perrando», Sanatorio «Frangioli. Salud 2000 SRL» Resistencia Chaco; Hospital Escuela «Gral. José Francisco de San Martín»

Insaurralde, M., Grandollo, P., Gómez, M., Servin R. (2009). Evaluación de los conocimientos y opiniones de médicos ante la prescripción de medicamentos genéricos en las provincias de Chaco y Corrientes. Revista de Posgrado de la Vía Cátedra de Medicina, No. 191.

Molina, M. (2011). Guia para la implementacion de estrategias de medicamentos genéricos en los paises de América Latina y el Caribe como mecanismo para mejorar el acceso a medicamentos. Organización Panamericana de la salud.

Ramírez, M. (2013). México avala a 33 nuevos medicamentos genéricos. El Economista.

UNED (2010). Marketing Farmacéutico. Tomos I, II, III y VI. Madrid: Universidad Nacional de Educación a Distancia. 